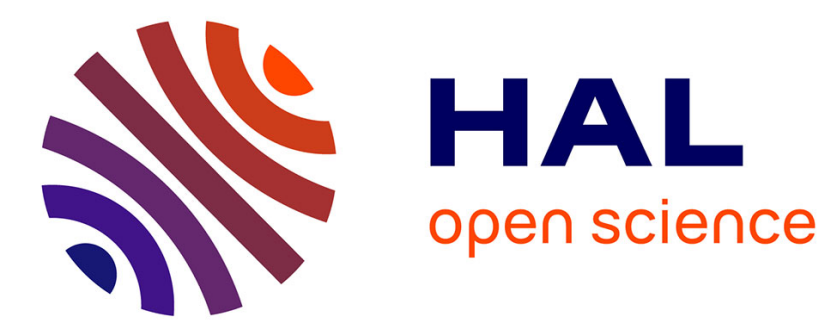

\title{
Hand-eye coordination relies on extra-retinal signals: Evidence from reactive saccade adaptation
}

\author{
Julien Cotti, Jean-Louis Vercher, Alain Guillaume
}

\section{To cite this version:}

Julien Cotti, Jean-Louis Vercher, Alain Guillaume. Hand-eye coordination relies on extra-retinal signals: Evidence from reactive saccade adaptation. Behavioural Brain Research, 2011, 218 (1), pp.248-252. 10.1016/j.bbr.2010.12.002 . hal-01384790

\author{
HAL Id: hal-01384790 \\ https://hal.science/hal-01384790
}

Submitted on 13 May 2018

HAL is a multi-disciplinary open access archive for the deposit and dissemination of scientific research documents, whether they are published or not. The documents may come from teaching and research institutions in France or abroad, or from public or private research centers.
L'archive ouverte pluridisciplinaire HAL, est destinée au dépôt et à la diffusion de documents scientifiques de niveau recherche, publiés ou non, émanant des établissements d'enseignement et de recherche français ou étrangers, des laboratoires publics ou privés. 


\title{
Hand-eye coordination relies on extra-retinal signals: Evidence from reactive saccade adaptation
}

\author{
Julien Cotti ${ }^{\mathrm{a}}$, Jean-Louis Vercher ${ }^{\mathrm{b}}$, Alain Guillaume ${ }^{\mathrm{a}, \mathrm{c}, *}$ \\ a Laboratoire de Neurobiologie de la Cognition, UMR CNRS 6155, Université de Provence, F-13331 Marseille, France \\ ${ }^{\mathrm{b}}$ Institut des Sciences du Mouvement, UMR CNRS 6233, Université de la Méditerranée, F-13288 Marseille, France \\ ' Fac. Sc. Sport, Université de la Méditerranée, F-13288 Marseille, France
}

\section{A R T I C L E I N F O}

\section{Article history:}

Received 15 September 2010

Received in revised form

30 November 2010

Accepted 5 December 2010

Available online 13 December 2010

\section{Keywords:}

Hand-eye coordination

Saccadic adaptation

Extra-retinal signals

Hand movement

Reactive saccade

\begin{abstract}
A B S T R A C T
Execution of a saccadic eye movement towards the goal of a hand pointing movement improves the accuracy of this hand movement. Still controversial is the role of extra-retinal signals, i.e. efference copy of the saccadic command and/or ocular proprioception, in the definition of the hand pointing target. We report here that hand pointing movements produced without visual feedback, with accompanying saccades and towards a target extinguished at saccade onset, were modified after gain change of reactive saccades through saccadic adaptation. As we have previously shown that the adaptation of reactive saccades does not influence the target representations that are common to the eye and the hand motor sub-systems (Cotti J, Guillaume A, Alahyane N, Pelisson D, Vercher JL. Adaptation of voluntary saccades, but not of reactive saccades. Transfers to hand pointing movements. J Neurophysiol 2007;98:602-12), the results of the present study demonstrate that extra-retinal signals participate in defining the target of hand pointing movements.
\end{abstract}

(c) 2010 Elsevier B.V. All rights reserved.

\section{Introduction}

When we move our hand to reach an object, we have usually previously produced a saccadic eye movement to align our line of sight to this object (for review, see [1-5]). This saccadic eye movement contributes to the accuracy of the reaching movement. The target location signal, initially noisy because being detected in retinal periphery, is indeed improved when the saccade aligns the fovea to the target. Additionally the sight of the hand at the end of the movement could participate in its guidance towards the target [6]. If the improvements in visual information generated by the accompanying saccades play a major role in hand movement control, other signals related to the oculomotor system may also be important in guiding the hand. The command generated for the eye movement and/or the eye position at the end of the saccade could in principle also be used for this guidance. Since they are non-related to vision, these signals are called extra-retinal signals. These extra-retinal signals coding gaze direction could contribute to the definition of a target for the hand.

In favor of a role of extra-retinal signals in guiding hand movements, several studies suggested that preventing eye motion, and

\footnotetext{
* Corresponding author at: Laboratoire de Neurobiologie de la Cognition, UMR CNRS 6155, Université de Provence, F-13331 Marseille, France. Tel.: +33 4135509 48; fax: +33488576872.

E-mail address: alain.guillaume@univmed.fr (A. Guillaume).
}

hence depriving hand sub-system of extra-retinal signals, lead to a decrease in hand movement accuracy [6-10]. Similarly, a relationship between mean amplitude of saccades and hand pointing movements (HPM) has also been reported (i) while varying saccade amplitude for a given required hand amplitude [11] or (ii) in the case of memorized targets [12]. However, in a more recent study, Desmurget et al. [13] found evidences against the hypothesis of a role of extra-retinal signals in HPM and concluded that "gaze direction does not serve as a target signal for arm motor guidance". These authors didn't observe the accuracy improvement when an eye saccade was allowed and also showed that an increase in gaze amplitude variability was not accompanied by a concomitant increase of hand amplitude variability. Also controversial is the existence of a correlation between eye and hand endpoints that could arise from a contribution of extra-retinal signals to the definition of a target for the hand. Some studies reported a significant correlation between the errors in pointing and in gaze at the time of pointing [14-17]. But other studies have reported this correlation to be very weak and inconsistent $[6,10,18-21]$.

The aim of the present study was to re-assess the putative role of extra-retinal signals in hand movement control using a novel approach. We used the progressive update of saccadic gain, termed "saccadic adaptation", to induce a perturbation in eye movements without the subject's knowledge [22]. Results of a previous study [23] form the basis of our reasoning. In the visuo-motor transformations involved in eye and hand visually directed movements, early representations of target location are common to both eye and 
hand motor sub-systems (e.g. in primary visual areas and probably in some later parietal areas). Cotti et al. [23] showed that adaptation of reactive saccades (RS) does not transfer to HPM produced in isolation (without accompanying saccade). This result entails that RS adaptation does not influence target representations common to the two visuo-motor transformations but regulates downstream structures (e.g. in the brainstem) specific to eye movements control. Hence, in a protocol with no visual feedback on hand performance and with target extinction at saccade onset (two precautions required to avoid the influence of visual information generated by the accompanying saccade), observing amplitude change of HPM produced with accompanying saccade after RS adaptation would necessarily entail that information related to the actual produced saccade (extra-retinal signals: efferent copy and/or ocular proprioception) influences HPM production.

Bekkering et al. [24] and Kröller et al. [25] previously studied the effect of saccadic adaptation on HPM accompanied by a saccade. Nevertheless, these studies were not designed to specifically assess the role of extra-retinal signals in hand-eye coupling and their protocols contained particularities that precluded firm conclusions on this question. First, Bekkering et al. [24] conducted a study on the effect of saccadic adaptation on HPM produced with accompanying saccades in a head restrained condition. The authors reported a strong influence of saccadic adaptation on HPM: 70\% of the change in saccade amplitude obtained through the adaptation procedure was observed for the hand movements. But in this study, adapted saccades were certainly of the voluntary type and we have recently demonstrated that voluntary saccade adaptation influences levels of target representation common to voluntary saccade and HPM $[23,26]$. The changes in HPM amplitude reported by Bekkering et al. [24] therefore likely reflected the change of some common target representation rather than an influence of extra-retinal signals on HPM (see [23]). Second, Kröller et al. [25] reported that HPM produced with an accompanying gaze saccade were shorter than baseline after the amplitude of RS saccades had been adaptively reduced. This result was nevertheless observed in a condition that allowed subjects to move their head during coordinated eye and hand movements. In a similar head-unrestrained condition in the same study, these authors also reported small but significant amplitude changes in hand movements that were tested without accompanying saccades after RS adaptation. Thus, in the head-unrestrained condition, RS adaptation could influence a target representation common to eye, head and hand movements. The change in hand movement amplitude observed when accompanying saccades were produced could therefore have resulted either from the modification of a common target signal or from the influence of extra-retinal signals. It is therefore impossible to draw conclusions about the role of extra-retinal signals using these data.

The present work was designed to evaluate the role of extraretinals signals in HPM avoiding the potentially confounding factors present in the Bekkering et al. and Kröller et al. studies: we measured, in a head-restrained condition, amplitude of HPM before and after having induced an amplitude decrease through saccadic adaptation of reactive saccades. Additional precautions were the absence of hand vision and of visual feedback after saccadic execution (target offset at saccade initiation). In these conditions, we found an amplitude change in HPM after adaptation of RS that unambiguously demonstrates that extra-retinal signals are involved in hand-eye coordination.

\section{Methods}

\subsection{Subjects}

Nine subjects volunteered to take part in the experiment. All were self-declared right-handed, 22-27 years of age ( mean $=23$ ) and healthy, with normal or correctedto-normal vision. They were naïve as to the purpose of the experiment. Prior to the

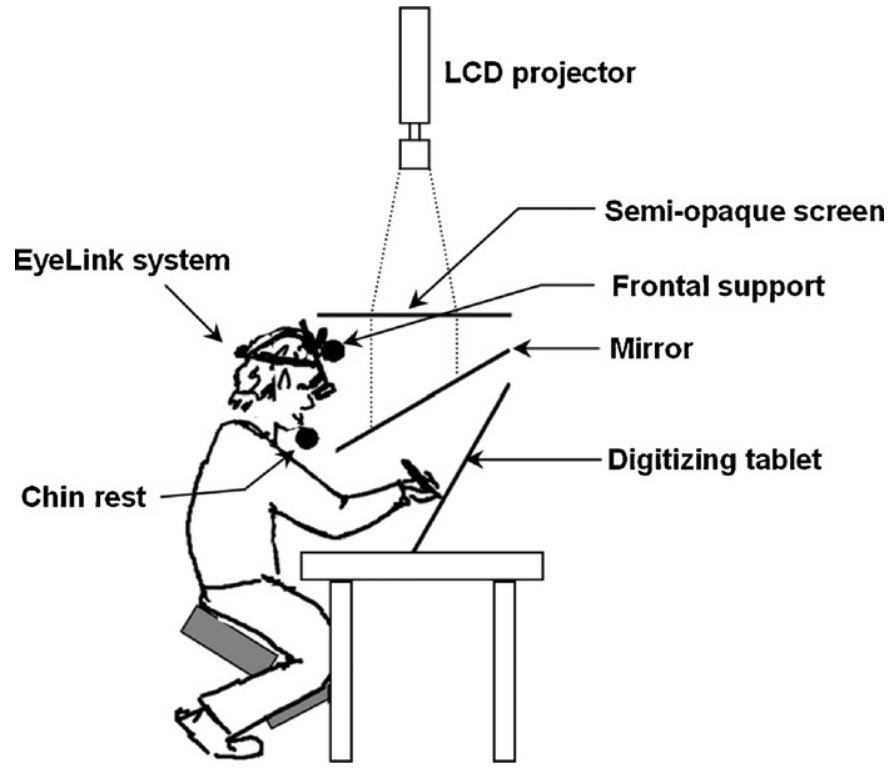

Fig. 1. Schematic representation of the experimental apparatus. Visual stimuli were projected onto a horizontal semi-opaque screen. Subjects faced an oblique mirror that reflected the images created on the semi-opaque screen. This setup created the illusion that visual targets were at the level of a third plane that corresponded to the surface of a large digitizing tablet.

experiment, subjects gave their informed consent to participate to the study, which was performed in accordance with the ethical standards laid down in the Declaration of Helsinki (last modified 2004).

\subsection{Apparatus}

The apparatus and experimental procedures in the present study were similar to those described in a previous paper [23]. Subjects were seated in complete darkness on an ergonomic posture chair (Fig. 1). The head was maintained straight ahead by a chin rest and a frontal support. Visual stimuli were projected (LCD projector) onto a horizontal semi-opaque screen. Subjects faced an oblique mirror which reflected the projected images. This setup created the illusion that visual targets were presented at the surface of a large digitizing tablet (SummaGrid IV, $0.1 \mathrm{~mm}$ accuracy, $100 \mathrm{~Hz}, 60 \times 90 \mathrm{~cm}$ ) placed underneath the mirror. As HPM were executed underneath the mirror, there was no visual feedback of hand movements (visual open-loop). The distance between the plane of the digitizing tablet and the subject's eyes was $57 \mathrm{~cm}$. A helmet-mounted infrared sensor allowed for recording of left eye position at $250 \mathrm{~Hz}$ (EyeLink video-oculographic system, SMI, Berlin, Germany) at a spatial resolution greater than $0.1^{\circ}$. The calibration was performed with the EyeLink software using a nine points calibration grid that allowed for precise measurements of horizontal and vertical eye position. A real-time acquisition system (Keithley Instruments, Cleveland, USA) controlled the experiments using laboratory-made software (Docometre). In particular, real-time monitoring of eye position allowed us to modify the visual display when the horizontal eye velocity exceeded a $30 \%$ threshold. Due to the visual display delay (12 ms), any display change (target offset in pre and post phases or target displacement in ADAPT phase) occurred approximately at saccadic peak velocity. This detection of movement onset was performed only for primary saccades, not for corrective saccades.

\subsection{Experimental procedure}

Eye and hand movements were directed towards two possible targets that appeared $20^{\circ}$ or $30^{\circ}$ to the left of a fixation point (FP). This FP was located $20^{\circ}$ to the right of the sagittal plane. Targets and the FP were presented on a black background and consisted of red circles (subtending $0.5^{\circ}$ of visual angle) with a black center to help foveation. An experimental session was composed of 6 phases (see Panel A of Fig. 2 for their temporal arrangement). HPM were produced with a stylus (held in the right hand) on the digitizing tablet and were accompanied by a leftward saccade (HPM/RS pre and post phases). To induce RS adaptation (RS ADAPT phase) and to evaluate the effect of this adaptation (RS pre and post phases), subjects were also required to produce leftward saccades in isolation. To take into account any potential saccadic de-adaptation during the HPM/RS post-adaptation phase, baseline RS amplitude evaluated before adaptation (RS pre) were compared to amplitude evaluated after RS adaptation, averaging values of two RS post-adaptation phases conducted just before (RS-post1) and after HPM/RS post-adaptation phase (RS-post2). 


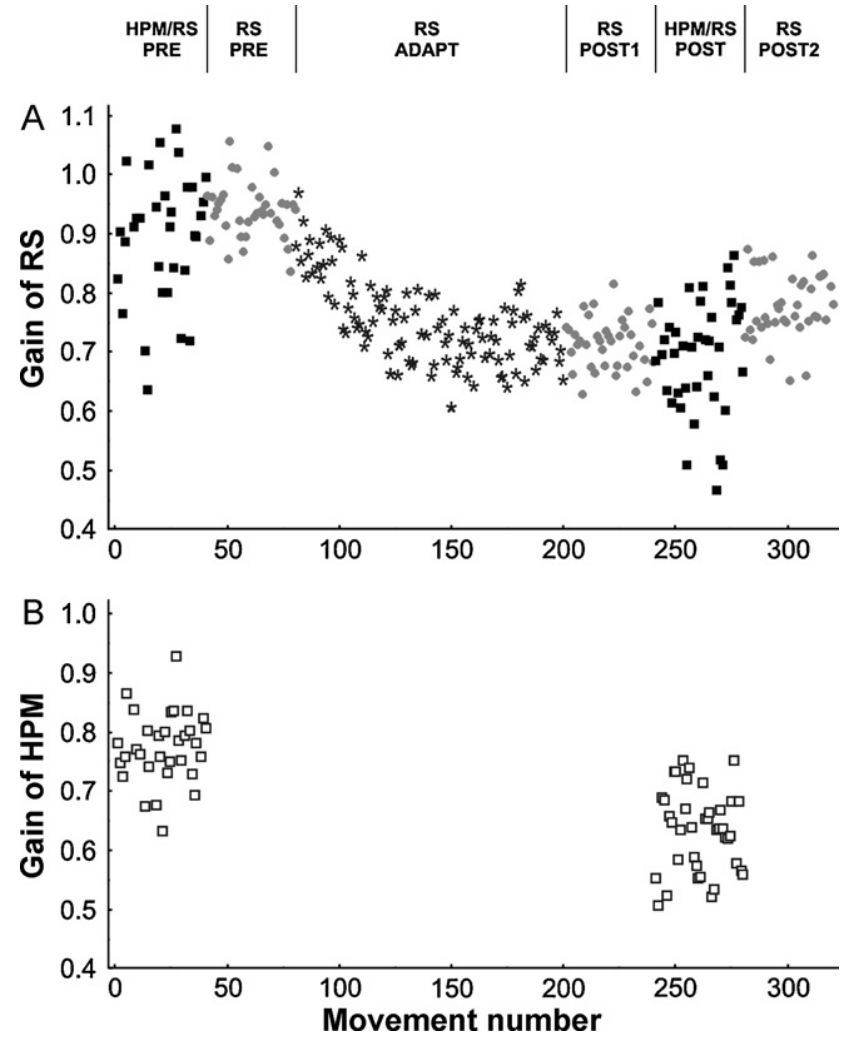

Fig. 2. Gain of RS and HPM for one representative subject. Each symbol corresponds to the amplitude of a single movement and is plotted as a function of movement number. (A) Black squares represent RS accompanying HPM. Grey circles and black stars represent RS produced in isolation during pre-and post-adaptation phases and during the adaptation phase, respectively. (B) Gain of HPM produced with RS.

Pre-adaptation, adaptation and post-adaptation trials had a constant duration (3 s). In HPM/RS-pre and HPM/RS-post phases, each trial started with the onset of the FP. Subjects were instructed to stare at the FP and to position the hand-held stylus within an L-shaped piece of wood sticked on the surface of the digitizing tablet. This setup did not hamper the subsequent leftward hand movement and ensured that the starting position of the stylus corresponded precisely to the visual FP. After a random period of time (600-1000 ms), the FP disappeared and one of the two targets was immediately presented. Subjects were asked to make a saccade and to point with the stylus towards this target. To avoid (i) the use of visual information concerning the target position with respect to the fovea and (ii) de-adaptation during post phase, the saccade onset triggered target extinction. No particular instruction was given concerning the onset of eye and hand movements: subjects were instructed to 'look and point in a natural manner, as quickly and accurately as possible'. Each pre- and post-adaptation phase consisted of 20 trials for each of the two target eccentricities, randomly distributed throughout the phase. The organization of RSpre and RS-post phases (design and number of trial, instruction for the subject) was identical to the one of HPM/RS phases but subjects were only required to produce saccades.

RS adaptation (RS ADAPT phase) was induced by the use of a classical doublestep paradigm [27]. Instructions for subjects were the same as in RS-pre and RS-post phases. Trial design was also the same except for two points. First, during the leftward saccade that was produced in response to the first target's step, the target shifted backwards (to the right) by an amount representing $30 \%$ of the initial target eccentricity (i.e., $6^{\circ}$ and $9^{\circ}$ respectively for $20^{\circ}$ and $30^{\circ}$ target eccentricity). Second, the target remained lit at the new position until trial-end. Due to the phenomenon of saccadic suppression $[28,29]$, the target's backward step remained unperceived by subjects. Because of this backward step, the first saccade 'missed' the target and an additional 'corrective' saccade was produced to foveate the new target position. Gradually, subjects unconsciously reduced the amplitude of the primary saccade, so that their gaze directly fell on the second target position at the end of the adaptation phase. RS-adaptation consisted of 120 trials (60 for each target eccentricity. randomly distributed throughout the phase).

\subsection{Data analysis}

For each trial, several characteristics (latency, duration and amplitude) of eye (all phases) and of hand (HPM/RS phases) movements were measured. To account for differences in the amplitude of saccadic and manual movements performed towards targets at different eccentricities $\left(20^{\circ} / 30^{\circ}\right)$, amplitude data were transformed into gains values. Gain was defined as the ratio between the amplitude of the primary saccadic or manual movement (before any corrective movement) and the eccentricity of the target relative to the FP. Comparisons between values (latency, duration and gain) obtained for the eye and the hand, before (pre) and after (post) the adaptation phase allowed assessing the effects of saccadic adaptation. These comparisons were performed through repeated measure ANOVAs with two factors $(2 \times 2)$ : Phase (Pre/Post) and Effector (Eye/Hand). Another repeated measure ANOVA with Phase (Pre/Post) and Condition (with HPM/without HPM) as factors allowed to evaluate the effect of the adaptation on saccades produced either with or without HPM. Statistical threshold was fixed to $P<0.05$.

\section{Results}

Concerning first the relative timing of eye and hand movements in HPM/RS phases, we found similar values to those observed in previous studies [9,30]: regardless of the experimental phase (i.e. pre- or post-adaptation), HPM onset occurred after the eyes had reached the target position, the target having been extinguished at saccade onset. In pre-adaptation phases, median latencies were $234 \mathrm{~ms}$ for the eye and $340 \mathrm{~ms}$ for the hand. Mean durations were $62 \mathrm{~ms}$ (eye) and $344 \mathrm{~ms}$ (hand). In post-adaptation phases, median latencies were $219 \mathrm{~ms}$ (eye) and $321 \mathrm{~ms}$ (hand) and mean durations were $56 \mathrm{~ms}$ (eye) and $320 \mathrm{~ms}$ (hand). Both ANOVAs for latency and duration resulted in a significant effect of the factor Effector $[F(1,8)=11.41(p<0.01)$ for Latency and $F(1,8)=557.85(p<0.001)$ for Duration], no effect of the factor Phase and no interaction between these factors.

As stated in the Introduction, several studies have already searched for a correlation between eye and hand endpoints when these movements are produced in visual open loop. Here the correlation analysis was conducted on individual subjects using eye gain and hand gain values (HPM/RS phases). In the pre-adaptation phase, none of the 9 subjects showed a statistically significant correlation between eye gain and hand gain. In the post-adaptation phase, only 2 out of 9 subjects showed a statistically significant correlation $(p<0.05)$, and this correlation was rather weak $(r=0.46$ and $r=0.4)$.

Concerning the effect of saccadic adaptation on movement gains, results obtained for a representative subject are presented in Fig. 2. RS and HPM gain values for the two target eccentricities, $20^{\circ}$ and $30^{\circ}$, were pooled together. The gain of RS without HPM (grey circles) significantly decreased from 0.94 (s.e.m. 0.02) before RS adaptation phase to 0.71 (s.e.m. 0.01) just after RS adaptation and 0.77 (s.e.m. 0.03) at the end of the experimental session (Fig. 2A). The gain decrease in RS induced by the double-step paradigm, computed as the difference between pre-adaptation RS gain and mean post-adaptation RS gain averaged over the two post-tests, reached 0.20 for this subject. This gain decrease corresponded to $66.6 \%$ of the 0.3 gain decrease that would have corresponded to full adaptation to the $30 \%$ backward step of the target. Similarly, the gain in RS with HPM (black squares) significantly decreased from 0.89 (s.e.m. 0.06) to 0.69 (s.e.m. 0.05) for this subject. The gain of HPM with $R S$ also significantly decreased from 0.79 (s.e.m. 0.04) before RS adaptation to 0.63 (s.e.m. 0.05) after RS adaptation (Fig. 2B).

A similar pattern of results was observed when all subjects were considered together. The double-step paradigm used in the present study induced a large gain change in both RS with HPM and RS without HPM after RS adaptation (for all the subjects) (Fig. 3A). The ANOVA used to compare the effect of the adaptation for these two conditions of saccade production resulted in a significant effect of the factor Phase $[F(1,8)=51.86(p<0.001)]$ without effect of the factor Condition $[F(1,8)=1.70(p>0.05)]$. But there was a significant interaction between these factors $[F(1,8)=8.35(p<0.05)]$ that corresponds to the fact that RS without HPM showed a larger gain decrease than RS with HPM [mean gains of RS with HPM: pre $=1.00$ (s.e.m. 0.03 ) vs. post $=0.80$ (s.e.m. 0.02 ); $66.7 \%$ of the required 

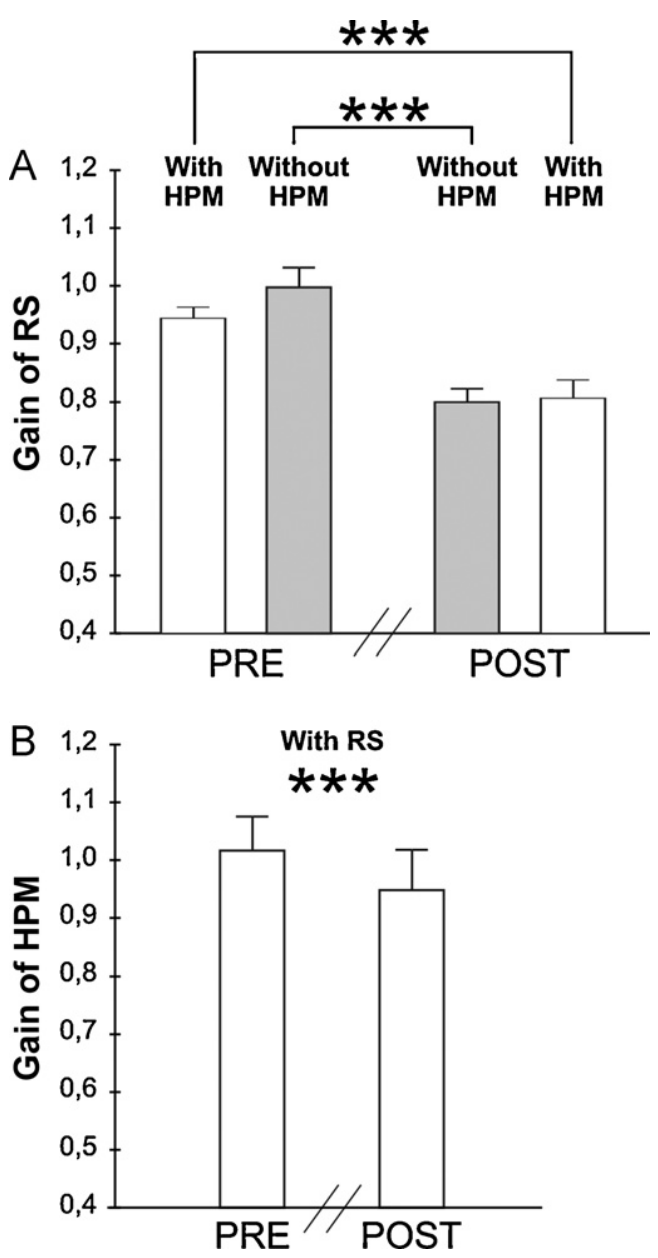

Fig. 3. Gain of RS (panel A) and HPM (panel B) before and after RS adaptation. Error bars correspond to standard errors of the mean $(n=9)$. Asterisks indicate the level of statistical significance of the factor Phase (Pre/Post) in ANOVA analyses $\left({ }^{* * *} p<0.001\right.$; see Section 3 for details). RS adaptation induced a change in the gain of HPM produced with accompanying saccades.

adaptation; mean gains of RS with HPM: pre $=0.94$ (s.e.m. 0.02) vs. post $=0.81$ (s.e.m. 0.03 ); $43.3 \%$ of the required adaptation]. A last ANOVA evaluated the effect of saccadic adaptation on RS with $H P M$ and on HPM. It resulted in a significant effect of the factor Phase $[F(1,8)=31.57(p<0.001)]$ but no effect of the factor Effector. In addition, no interaction was found between theses factors that indicates that there was a congruent decrease of RS with HPM and of HPM. Mean HPM gains were 1.02 (s.e.m. 0.06) in pre and 0.95 (s.e.m. 0.07) in post phases. This HPM gain decrease represents $35 \%$ of the gain change recorded for RS without HPM (i.e. the amount of adaptation reached with the RS ADAPT phase).

\section{Discussion}

The aim of this study was to further investigate the role of extraretinal signals in hand-eye coordination. After having adaptively reduced the gain of reactive saccades (RS), we observed a congruent gain reduction in hand pointing movements (HPM) performed with accompanying RS. Since we previously demonstrated that RS adaptation does not modify stages of target representation that are common to the eye and the hand motor sub-systems [23], HPM gain reduction was necessarily due to an influence of information related to the actually produced eye saccade. Since HPM were executed in the absence of visual feedback concerning hand position and towards targets that were extinguished at saccade onset, these information related to the produced saccade could only consist in extra-retinal signals.

The two previous studies of Bekkering et al. [24] and Kröller et al. [25] that also tested the effect of saccadic adaptation on HPM with accompanying saccades contained protocol features that preclude a firm conclusion concerning the influence of extra-retinal signals (see Introduction). A third study may also be considered here: Bruno and Morrone [31] observed a significant effect of saccadic adaptation on verbal or pointing reports, both produced after the completion of a saccade. Can their result concerning pointing reports could also be explained by an influence of extra-retinal signals related to the produced saccade? We believe the answer is no for the following reasons. First, in their study eye and hand movements were dissociated both in time and space. The saccade was produced well before the HPM and was directed towards the target used for the adaptation whereas the HPM was directed towards another target (a probe used to evaluate the effect of adaptation on nearby locations). Second, these authors observed an effect of saccadic adaptation on verbal reports, also performed after the production of a saccade. This effect is most likely due to the fact that when a probe used for a perceptual judgement is flashed while subjects prepare a saccade towards an 'adapted' target, the metrics of the saccade which would be necessary (though actually not initiated) to foveate the probe contribute to its spatial localization. This results in a mislocalization field similar to the well known adaptation fields [32,33]. In the study of Bruno and Morrone, both effects on verbal and pointing reports have similar spatial pattern around the adapted target. This suggests that pointing reports were prone to a false perception rather than being influenced by extra-retinal signals.

If extra-retinal signals help guiding the hand, which is demonstrated here, a correlation between eye and hand end-points is likely to be observed. Nevertheless, as stated in the Introduction, the existence of this correlation is debated. In the present study no correlation was found between eye and hand gains (and by extension, between endpoints of saccadic and manual movements since starting positions were fixed). A potential explanation to this negative result may be linked to the presence of high levels of noise in these motor systems that would hide this correlation. Sailer et al. [21] suggested that potential spatial coupling could be masked by a high rate of motor noise occurring independently in unshared parts of the motor systems during movement execution. Independent noise appearing at a hierarchically low level, i.e. downstream from the influence of extra-retinal signals, during motor generation could interfere with potential coupling of both motor systems. The methodology used in the present study, relying on the mean of many trials, may have been more sensitive to reveal this link between the eye and hand movements.

Our study demonstrates that extra-retinal signals play a role in hand-eye coordination. Is it possible to favor the efference copy or the ocular proprioception in this influence of saccade related information on HPM? Several lines of evidence are in favor of the efference copy hypothesis. Wilmut et al. [34] tested the effect of producing saccades on the accuracy of sequential pointing and concluded that the role of ocular proprioception was far less important than the role of the feedforward system that is activated when an eye movement is initiated. Van Donkelaar et al. [35] provided even more direct evidence. The influence of decorrelating eye and hand movement start points (see Section 1) on hand movement amplitude is significantly reduced by transcranial magnetic stimulation over the posterior parietal cortex when stimulation is delivered $0-100 \mathrm{~ms}$ prior to saccade onset. This result strongly suggests that the hand-eye interaction depends on integration of the efferencecopy of the produced saccade by the hand motor system rather than on integration of extra-oculomotor muscles proprioceptive information. Altogether, these results render it very likely that the 
decrease in HPM amplitude after saccadic adaptation reflects the reading of the saccadic efference-copy by the hand control system. If we accept the efference-copy hypothesis, results of the present study have implications regarding mechanisms of RS adaptation. Their adaptation necessarily occurs upstream or at the level of the production of this efference-copy. Indeed, after the adaptation phase, only the use of a modified efference-copy could lead to a change in HPM amplitude congruent with the change in saccadic amplitude.

Finally, the present study was designed to address the question of an influence of eye movements (and the related extra-retinal signals) on hand movements, but a reverse interaction from the arm to the eye is also possible. For example, it has been demonstrated that saccades show a small but significant increase in peak velocity when eye and hand movements are directed to the same target $[36,37]$. In addition, there is neurophysiological evidences for an effect of arm movement command production in oculomotor structures [38,39]. In the present study, gathered data also revealed this reverse interaction, i.e. an influence of arm movement on saccadic eye movements. First, we also observed a small increase in peak velocity for saccades accompanying hand movements (data not presented here). Second, the reduction in gain for saccades accompanying hand movements was smaller than for saccades produced in isolation. This latter result could be accounted by two hypotheses: (i) Signals associated with the hand planning mute the adaptive effects observed in the saccadic system; (ii) If we consider data before adaptation, saccades produced with HPM have smaller gain. This tendency of smaller saccades when accompanied by a HPM has recently been described in the monkey [40]. There would be a disappearance of this tendency after saccadic adaptation. Whatever the explanation considered, the present results are another evidence in favor of an influence of HPM on saccadic eye movements.

In conclusion, the possibility that extra-retinal signals contribute to the definition of a target for hand pointing movements remains debated. The present study demonstrates that hand pointing movements produced towards a target that is switched off long before their initiation, and performed in visual open loop, are influenced by the characteristics of accompanying saccades. Extraretinal signals generated during reactive saccade production thus participate in the guidance of hand movements.

\section{Acknowledgements}

We thank J.T. Coull for helpful reading of this manuscript. JC, JLV were supported by CNRS. AG was supported by Université de la Méditerranée.

\section{References}

[1] Bekkering H, Sailer U. Commentary: coordination of eye and hand in time and space. In: Hyona J, editor. The brain's eye: neurobiological and clinical aspects of oculomotor research. Prog Brain Res. Elsevier; 2002. p. 365-73.

[2] Crawford JD, Medendorp WP, Marotta JJ. Spatial transformations for eye-hand coordination. J Neurophysiol 2004;92:10-9.

[3] Desmurget M, Pélisson D, Rossetti Y, Prablanc C. From eye to hand: planning goal-directed movements. Neurosci Biobehav Rev 1998;22:761-88.

[4] Pelisson D, Prablanc C. Eye-hand coordination. In: Binder MD, Hirokawa N, Windhorst U, editors. Encyclopedia of neuroscience. Heidelberg, Germany: Springer; 2008. p. 1540-2.

[5] Prablanc C, Desmurget M, Grea H. Neural control of on-line guidance of hand reaching movements. In: Prablanc $C$, editor. Neural control of space coding and action production. Prog Brain Res. Elsevier; 2003. p. 155-70.

[6] Prablanc C, Echallier JF, Komilis E, Jeannerod M. Optimal response of eye and hand motor systems in pointing at a visual target 1 . Spatio-temporal characteristics of eye and hand movements and their relationships when varying the amount of visual information. Biol Cybern 1979;35:113-24.

[7] Abrams RA, Meyer DE, Kornblum. Eye-hand coordination: oculomotor control in rapid aimed limb movements. J Exp Psychol - Hum Percept Perform 1990; 16:248-67.
[8] Henriques DYP, Klier EM, Smith MA, Lowy D, Crawford JD. Gaze-centered remapping of remembered visual space in an open-loop pointing task. J Neurosci 1998;18:1583-94.

[9] Vercher JL, Magenes G, Prablanc C, Gauthier GM. Eye-head-hand coordination in pointing at visual targets: spatial and temporal analysis. Exp Brain Res 1994;99:507-23.

[10] Delreux V, Vandenabeele S, Crommelinck M, Roucoux A. Interactions between goal-directed eye and arm movements - arguments for an interdependent motor control. J Mot Behav 1991;23:147-51.

[11] Van Donkelaar P. Eye-hand interactions during goal-directed pointing movements. Neuroreport 1997;8:2139-42.

[12] Enright JT. The non-visual impact of eye orientation on eye-hand coordination. Vision Res 1995;35:1611-8.

[13] Desmurget M, Turner RS, Prablanc C, Russo GS, Alexander GE, Grafton ST. Updating target location at the end of an orienting saccade affects the characteristics of simple point-to-point movements. J Exp Psychol - Hum Percept Perform 2005;31:1510-36.

[14] Admiraal MA, Keijsers NLW, Gielen CCAM. Interaction between gaze and pointing toward remembered visual targets. J Neurophysiol 2003;90: 2136-48.

[15] Gielen CCAM, Heuvel PJM, Gisbergen JAM. Coordination of fast eye and arm movements in a tracking task. Exp Brain Res 1984;56:154-61.

[16] Flanders M, Daghestani L, Berthoz A. Reaching beyond reach. Exp Brain Res 1999;126:19-30.

[17] Medendorp WP, Crawford JD. Visuospatial updating of reaching targets in near and far space. Neuroreport 2002;13:633-6.

[18] Biguer B, Jeannerod M, Prablanc C. The coordination of eye, head, and arm movements during reaching at a single visual target. Exp Brain Res 1982;46:301-4.

[19] Henriques DYP, Medendorp WP, Gielen CCAM, Crawford JD. Geometric computations underlying eye-hand coordination: orientations of the two eyes and the head. Exp Brain Res 2003;152:70-8.

[20] Ma-Wyatt A, Stritzke M, Trommershäuser J. Eye-hand coordination while pointing rapidly under risk. Exp Brain Res 2010;203:131-45.

[21] Sailer U, Eggert T, Ditterich J, Straube A. Spatial and temporal aspects of eye-hand coordination across different tasks. Exp Brain Res 2000;134: 163-73.

[22] Hopp JJ, Fuchs AF. The characteristics and neuronal substrate of saccadic eye movement plasticity. Prog Neurobiol 2004;72:27-53.

[23] Cotti J, Guillaume A, Alahyane N, Pelisson D, Vercher JL. Adaptation of voluntary saccades, but not of reactive saccades. Transfers to hand pointing movements. J Neurophysiol 2007;98:602-12.

[24] Bekkering H, Abrams R.A., Pratt J. Transfer of saccadic adaptation to the manual motor system. Hum Mov Sci 1995;14:155-64.

[25] Kröller J, De Graaf JB, Prablanc C, Pelisson D. Effects of short-term adaptation of saccadic gaze amplitude on hand-pointing movements. Exp Brain Res 1999; $124: 351-62$

[26] Cotti J, Panouilleres M, Munoz DP, Vercher JL, Pelisson D, Guillaume A. Adaptation of reactive and voluntary saccades: different patterns of adaptation revealed in the antisaccade task. J Physiol 2009;587:127-38.

[27] Mclaughlin SC. Parametric adjustment in saccadic eye movements. Percept Psychophys 1967;2:359-61.

[28] Bridgeman B, Hendry D, Stark L. Failure to detect displacement of the visual world during saccadic eye movements. Vision Res 1975;15:719-22.

[29] Matin E. Saccadic suppression: a review and an analysis. Psychol Bull 1974;81:899-917.

[30] Prablanc C, Echallier JE, Jeannerod M, Komilis E. Optimal response of eye and hand motor systems in pointing at a visual target 2 . Static and dynamic visual cues in the control of hand movement. Biol Cybern 1979;35:183-7.

[31] Bruno A, Morrone MC. Influence of saccadic adaptation on spatial localization: comparison of verbal and pointing reports. J Vis 2007;7(5), 16.1-13.

[32] Collins T, Dore-Mazars K, Lappe M. Motor space structures perceptual space evidence from human saccadic adaptation. Brain Res 2007;1172:32-9.

[33] Schnier F, Zimmermann E, Lappe M. Adaptation and mislocalization fields for saccadic outward adaptation in humans. J Eye Mov Res 2010;3(3):1-18.

[34] Wilmut K, Wann J, Brown J. How active gaze informs the hand in sequential pointing movements. Exp Brain Res 2006;175:654-66.

[35] Van Donkelaar P, Lee JH, Drew AS. Transcranial magnetic stimulation disrupts eye-hand interactions in the posterior parietal cortex. J Neurophysiol 2000;84:1677-80.

[36] Epelboim J, Steinman RM, Kowler E, Pizlo Z, Erkelens CJ, Collewijn H Gaze-shift dynamics in two kinds of sequential looking tasks. Vision Res 1997;37:2597-607.

[37] Snyder LH, Calton JL, Dickinson AR, Lawrence BM. Eye-hand coordination: saccades are faster when accompanied by a coordinated arm movement. J Neurophysiol 2002;87:2279-86.

[38] Stuphorn V, Bauswein E, Hoffmann KP. Neurons in the primate superior colliculus coding for arm movements in gaze-related coordinates. J Neurophysiol 2000;83:1283-99.

[39] Werner W. Neurons in the primate superior colliculus are active before and during arm movements to visual targets. Eur J Neurosci 1993;5: 335-40.

[40] Kattoulas E, Smyrnis N, Mantas A, Evdokimidis I, Raos V, Moschovakis A. Arm movement metrics influence saccade metrics when looking and pointing towards a memorized target location. Exp Brain Res 2008;189: 323-38. 